\title{
Musings on Multiplication Tables and Associated Mathematics and Teaching Practices
}

\author{
Faaiz Gierdien \\ Research Unit for Mathematics Education, University of Stellenbosch \\ faaiz@sun.ac.za
}

\begin{abstract}
This paper is based on my reflections on a deceptively simple tabular representation of a combined $12 \times 12$ multiplication table showing multiplier and multiplicand, starting at a time when I taught mathematics full time at a primary (elementary) school through to my present teaching of mathematics education modules to prospective teachers. A historically-motivated framework on the importance of tables as expressions of complex information in two-dimensional form is used to gain insight into and understand multiplication tables. Through this framework it is shown that the modal practice of "knowing one's tables" in the primary grade levels is really about knowing sequenced and separated lists of whole number multiplications. In contrast, tabular multiplication sequences in a combined multiplication table can, through appropriate teaching practices, enable the discovery of multiple relationships beyond multiplication or arithmetic, resulting in significant mathematics that spans the grade levels. Implications for mathematics teacher education practice with its current focus mathematical knowledge for teaching, are considered.
\end{abstract}

Beyond the so-called multiplication tables there is significant mathematics in a $12 \times 12$ tabular representation of the multiplication tables (see Figure 1), which deserves attention. "What" this mathematics is has a great deal to do with "how" one approaches Figure 1. On a similar point, Dewey (1904/1964) writes extensively on dichotomies such as content and pedagogy, subject matter and method, and the implication of separating the two. Put differently, "what" the mathematics in Figure 1 is as content or subject matter has to do with how or the pedagogy or method that is used. This is the main argument for the title of the paper, namely mathematics and teaching practices.

We know only too well the inert status of the multiplication tables in relation to the rest of the mathematics curriculum. The idea of the paper is therefore to throw multiplication tables into "fresh combinations" (Whitehead, 1929/1951) with other branches of the mathematics curriculum. Any curriculum associated with Figure 1 is thus intimately connected to the type of teaching that accompanies it.

In this article I reflect on some of the mathematics and teaching practices associated with Figure 1 which I first noticed in Burns (2000) while teaching mathematics full time at an elementary (primary) school. I became intrigued by the structure, pattern and symmetry in this multiplication table and the attendant history of tables as human inventions and have been using it since in prospective mathematics education modules and professional development opportunities for in-service teachers.

Current curriculum policy statements in South Africa provide a certain orientation towards multiplication tables that is helpful but also inadequate (Department of Education (DOE), 2003). In the primary or elementary grade levels, multiplication is mainly associated with the multiplication tables, hence there will be a focus on references to multiplication in the policy document (DOE, 2003). Policy statements contain no detailed vignettes that illustrate the power of tables with respect to multiplication tables to start with. For example, they suggest that multiplication should be introduced through "repeated addition" in 
the first grade and then multiplication of whole 1-digit by 1-digit numbers in the second grade and whole 2 digit by 1-digit numbers in the third grade. From the fourth to the sixth grades learners should be encouraged to memorise multiplication fluently to at least $12 \times 12$. The point to note is that multiplication tables, through an understanding of tables per se is never revisited further along in the grade levels in terms of their potential to connect multiplication tables with other mathematics. Moreover, confining the multiplication tables to Learning Outcome 1 - numbers, operations and relationships - is a limiting conception of the possible power of tables as bearers of mathematical information. Depending on the representations of multiplication tables that are being used and accompanying investigations as suggested in the policy document, it can be shown too, that they connect with Learning Outcome 2 - patterns, functions and algebra, for example. Such investigations can take us well into grade levels 11 through 12, i.e. the Further Education and Training band starting with the multiplication tables in the lower grade levels.

\begin{tabular}{|c|c|c|c|c|c|c|c|c|c|c|c|c|}
\hline$\times$ & $\mathbf{1}$ & $\mathbf{2}$ & $\mathbf{3}$ & $\mathbf{4}$ & $\mathbf{5}$ & $\mathbf{6}$ & $\mathbf{7}$ & $\mathbf{8}$ & $\mathbf{9}$ & $\mathbf{1 0}$ & $\mathbf{1 1}$ & $\mathbf{1 2}$ \\
\hline $\mathbf{1}$ & 1 & 2 & 3 & 4 & 5 & 6 & 7 & 8 & 9 & 10 & 11 & 12 \\
\hline $\mathbf{2}$ & 2 & 4 & 6 & 8 & 10 & 12 & 14 & 16 & 18 & 20 & 22 & 24 \\
\hline $\mathbf{3}$ & 3 & 6 & 9 & 12 & 15 & 18 & 21 & 24 & 27 & 30 & 33 & 36 \\
\hline $\mathbf{4}$ & 4 & 8 & 12 & 16 & 20 & 24 & 28 & 32 & 36 & 40 & 44 & 48 \\
\hline $\mathbf{5}$ & 5 & 10 & 15 & 20 & 25 & 30 & 35 & 40 & 45 & 50 & 55 & 60 \\
\hline $\mathbf{6}$ & 6 & 12 & 18 & 24 & 30 & 36 & 42 & 48 & 54 & 60 & 66 & 72 \\
\hline $\mathbf{7}$ & 7 & 14 & 21 & 28 & 35 & 42 & 48 & 56 & 63 & 70 & 77 & 84 \\
\hline $\mathbf{8}$ & 8 & 16 & 24 & 32 & 40 & 48 & 56 & 64 & 72 & 80 & 88 & 96 \\
\hline $\mathbf{9}$ & 9 & 18 & 27 & 36 & 45 & 54 & 63 & 72 & 81 & 90 & 99 & 108 \\
\hline $\mathbf{1 0}$ & 10 & 20 & 30 & 40 & 50 & 60 & 70 & 80 & 90 & 100 & 110 & 120 \\
\hline $\mathbf{1 1}$ & 11 & 22 & 33 & 44 & 55 & 66 & 77 & 88 & 99 & 110 & 121 & 132 \\
\hline $\mathbf{1 2}$ & 12 & 24 & 36 & 48 & 60 & 72 & 84 & 96 & 108 & 120 & 132 & 144 \\
\hline
\end{tabular}

Figure 1: A combined multiplication table

Teaching investigations that are fruitful and beyond multiplication tables imply instructing students in ways that draw attention to the myriad of patterns in the rows, columns and diagonals in Figure 1 and the questions we pose about them. This Deweyan idea of the "what" and the "how" relates to a current focus in mathematics teacher education research, namely "mathematics for teaching" (Adler, 2005; Ball \& Bass, 2000; Ball, Bass, \& Hill, 2004). In a sense we can think of the "what" as mathematics and the "how" as teaching. When it comes to teaching, the mathematics for teaching literature alerts us to compression and decompression or unpacking of the mathematics in Figure 1.

For the purposes of this paper, teaching practices refer to teaching and learning situations exemplified by the uses and non-uses of mathematical skills such as specialising, generalising, representing, problem posing and recognising and extending patterns (DOE, 2003) in relation to Figure 1 or otherwise stated. When teachers teach the multiplication tables, they instruct their students in various ways. Some might focus on a rote memorisation while others might want their students to search for patterns with respect to odd or even numbers when they are multiplied. Yet others will want their students to see beyond the multiplication and will push them to ask what happens if? or how can we generalise a diagonal or a horizontal sequence?, thereby focusing on structure in their teaching. Amongst others, Mason's (1998), Brown and Walter's (1990) and Olivier's $(1999 ; 2002)$ work exemplify these mathematical skills.

It should be noted that mathematics for teaching with respect to Figure 1 can also be connected to multiplication literature and other literature on ways of connecting arithmetic and algebra. These are instances of fresh combinations. The first implies paying attention to Steffe's (1988; 1994), Izsák's (2004; 2005) and Clark and Kamii's (1996) work on the importance of fostering and identifying children's 
multiplication schemes, through the use of multiple representations and particular mathematical skills involving investigations. The same holds true for the second, where we bridge the "gap" between arithmetic and algebra (Blanton \& Kaput, 2003; Nunn, 1919; Olivier, 1999; 2002; Sfard \& Linchevski, 1994). For instance, it can be shown that through particular investigations, algebra can come about through the reification or generalisation of arithmetical processes noticeable in possible recursive and functional relationships of the arithmetical equivalences in Figure 1. If this is done we notice the emergence of mathematical or algebraic objects at another level, namely arithmetical processes becoming compressed as symbols.

Such type of investigations with respect to Figure 1 are important for prospective and in-service teachers because they are about ways of seeking alignment with the policy statements on learning outcomes and mathematical skills and to mathematics for teaching. Concerning prospective teachers with whom I am working presently, Adler (2005, p. 5) asks where and how prospective teachers are provided with opportunities for learning to unpack mathematics, and so develop pedagogically useful mathematics, i.e. mathematics for teaching. As a first step, we need to loosen up earlier and present iterations of meanings and thereby subtly question settled dogmas such as knowing one's tables.

\section{A historical perspective on tables, multiplication tables and related teaching practices}

The construction of Figure 1 has to be viewed in light of the historical legacy of tables as bearers of information or data in two dimensions. The construction of tables and tabular formatting occurred in ancient Iraq during the second millennium before the Common Era. It is the best documented period of mathematics in scribal schools (Robson, 2000) and thus an appropriate period to look at for the purposes of this paper, which in part focuses on school mathematics. During this period, scribes developed the "tabular account" as an efficient way of sorting, recording and storing data (Robson, 2003). Some of the data they worked with were in the area of metrology, e.g. weights, areas, and volumes and capacities. Here they used tables to do conversions between different weight, area and volume units, for example.

This is of course not the only purpose of tables. A key feature of the tabulation that evolved then is the horizontal and vertical separation of quantitative and sometimes qualitative data such as the number of sheep in a flock, or areas and capacities, for example (Robson, 2003). A table thus has rows and columns that are aligned. It could also be linear, i.e. having columns only that function more like columns of a newspaper than columns of a table. In such a case there are no relationships between the information or data in rows and in columns and we have a "list." In such a case it would not be possible to find or express numerical or other relationships between and within columns and rows. This distinction between tables and lists must be borne in mind when we examine multiplication tables in present day schools.

There is more to know about multiplication lists. We now know that some multiplication or arithmetical lists during this period in Babylonia were "prosaic," or verbose, i.e. in words, while others were terse. Robson (1998) gives the example of a tablet of the 2 times tables (see Figure 2):

\begin{tabular}{llll}
\hline Obverse & & Reverse \\
\hline 2 times 1 & 2 & times 12 & 24 \\
times 2 & 4 & etc. & $\ldots$ \\
times 3 & 6 & $\ldots$ & $\ldots$ \\
$\ldots$ & & $\ldots$ & $\ldots$ \\
$\ldots$ & & $\ldots$ & $\ldots$ \\
$\ldots$ & & $\operatorname{times~30}$ & 100 \\
$\ldots$ & & $\operatorname{times~} 40$ & 120 \\
times 11 & 22 & $\operatorname{times} 50$ & 140 \\
\hline
\end{tabular}

Figure 2: The 2 times tables taken from the Babylonian period 
These would be the 2 times tables in the sexagesimal place value system, explaining why 2 times 40 equals 120 ! These multiplication tables are verbose because of the use of words ("times"). In terms of teaching, students in scribal schools were required to memorise a list of a standard series of multiplication through repeated copying and revision. They would do this first as individual sections and then as long extracts from the whole list (Robson, 2003, p. 31), which could be up to 1000 lines in total! Robson (2003, pp. 32-36) shows the example of a fifteen times multiplication table, meaning that she questions our use of table. She notes that our collective blindness to document formatting has caused us to refer erroneously to the intrinsically tabular documents as "lexical lists" in Sumerian and Akkadian translations for example, while their list-like arithmetical counterparts are always known as "multiplication tables" (emphasis in original). It is therefore not surprising that in some of her research she calls tables a misnomer because the scribal students were in fact memorising lists of number facts (Robson, 2002, p. $361)$.

More intriguing is that she found a strong disinclination towards a truly tabular format in school arithmetic and thus in its teaching uses. Here one might have expected the proliferation of tables! She notes that Mesopotamian scholastic mathematics employed tables very rarely, preferring to express arithmetical and metrological equivalences as lists (Robson, 2003, p. 42). Truly tabular mathematical documents at the time have their debt to administrative and astronomical practices. In other words, such documents have their rows and columns aligned, i.e. they have a two-dimensional layout or format from which data such as the visibility of planets or stars and time of year or month can be retrieved because of the two dimensional format.

In her more recent work, she observes that we underestimate the invention of tables as "truly powerful information-processing tools, cognitively distinct from well-organised lists" (Robson, 2007, p. 421). In this sense Figure 1 is not a list but a "truly tabular table" (Robson, 2003, p. 42) because the reader's cognition or attention can be directed to the myriad of relationships between the numbers in the rows, columns and diagonals. These turn out to be of a profound nature, which we can investigate depending on the problems or questions we pose about them. For example, we can investigate the numerical information imbedded in Figure 1 beyond multiplication, through the use of the mathematical skills mentioned earlier, thereby opening up the reader's recognition as to what the relationships could be.

We should be careful not to dismiss the Babylonian period in terms of its school mathematics as being simply a period of multiplication lists accompanied by teaching practices that focused on a rote memorisation of multiplication facts in sequence, and a laborious copying of long lists of multiplication in verbose and terse forms. Instead, it is the cognitive distinction between columnar and row organisation in the construction of Figure 1, which has its precedent in old Babylonian tables stemming in part from tabular bookkeeping or accounting for instance, that we should appreciate (Robson, 2004).

To summarise, Robson's work points us to the need to differentiate between lists and tables or list-like and truly tabular representations, and hence multiplication lists and tables. The former are onedimensional, typical of newspaper columns, whereas tables essentially have a two-dimensional layout where numerical or other type of information is stored, recorded and from whence it can be retrieved. It is with this lens that we now turn our attention to multiplication tables and related teaching practice in schools.

\section{On modal multiplication tables teaching practice in schools}

The format or layout of multiplication tables in schools and the associated teaching practice impact on what mathematics students learn and in turn how they learn. In most schools, multiplication tables are represented as separate albeit well-organised lists of arithmetical equivalences for the different multipliers 1 through 12. A reason for 12 may well have to do with dozens, pennies and inches. From prospective and in-service teachers' anecdotes and what I have observed, these representations are usually in the form of wall charts, as one teacher reported during a professional development opportunity connected to a mathematics teacher education project (Figure 3): 


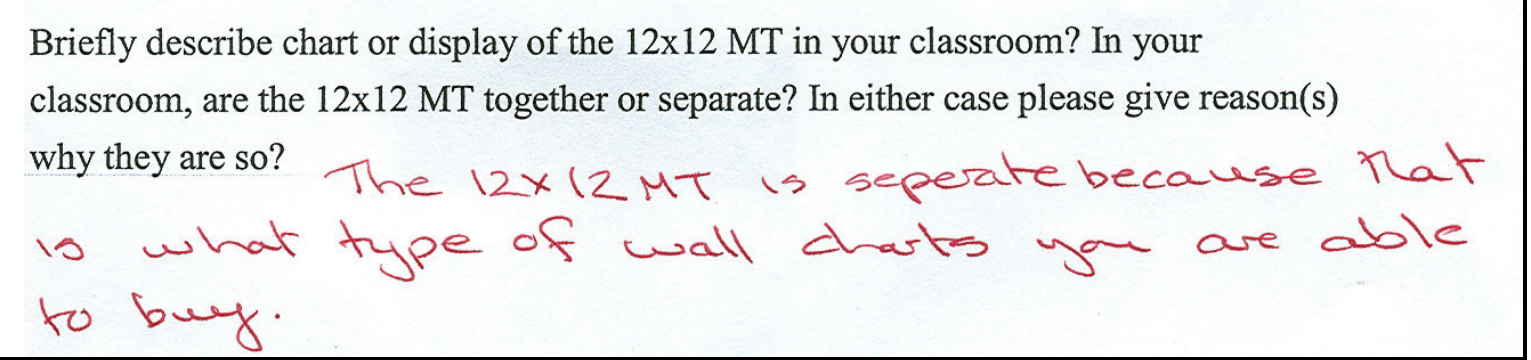

Figure 3: A teacher's explanation for the $12 \times 12$ multiplication tables (MT) in classrooms

Such wall charts are no doubt well-organised and in sequence, meaning arithmetic equivalences or "multiplication facts" are listed from 1 through 12. This is probably no different from what Robson (1998; 2002; 2003) found in the case of scholastic mathematics in Mesopotamia. Through systematic and careful teaching, students' cognition could be directed towards patterns and structures that are separate for each of the multipliers and multiplicands. These would be confined to the columns that one needs to read in a vertical manner, such as newspaper columns. This newspaper-like representation in wall charts does not afford students or teachers the opportunity to make cognitive distinctions between columnar and row organisation as is possible in Figure 1. In the latter it is possible to pose questions about the rows and diagonals as will be seen later on in this paper. It is the two-dimensionality of tables per se that is missing in separately listed multiplication equivalences as in the wall charts described by the teacher.

What is thus evident is that the layout of multiplication tables in schools impacts on teaching. Sources of evidence for this claim are my own schooling and the anecdotal accounts given by prospective and practicing teachers in the mathematics education modules I teach for the past several years. Most of my current students have never encountered a multiplication table like the one in Figure 1. They describe multiplication tables in schools as separate lists for each of the different multipliers 1 through 12 , including zero at times.

In terms of teaching practices they paint pictures of moments where they have had to sit in corners and memorise the "difficult' "seven times tables" and "twelve times tables." They were surprised to see connections between the ten times tables and the two times tables through the distributive property in the case of the twelve times tables. Thus, what we have in most schools are well-organised multiplication lists in sequence as opposed to truly tabular multiplication tables such as Figure 1. In our teaching practice are we insisting that our students know their multiplication tables or their multiplication lists?

By examining instances of teaching practice with regards to multiplication tables we gain a clearer sense of what students, whether children and/or prospective teachers are learning and also how they are learning. Figure 4 shows a reproduction of an abbreviated version of commercially available worksheets on multiplication tables aimed at the middle grade levels:

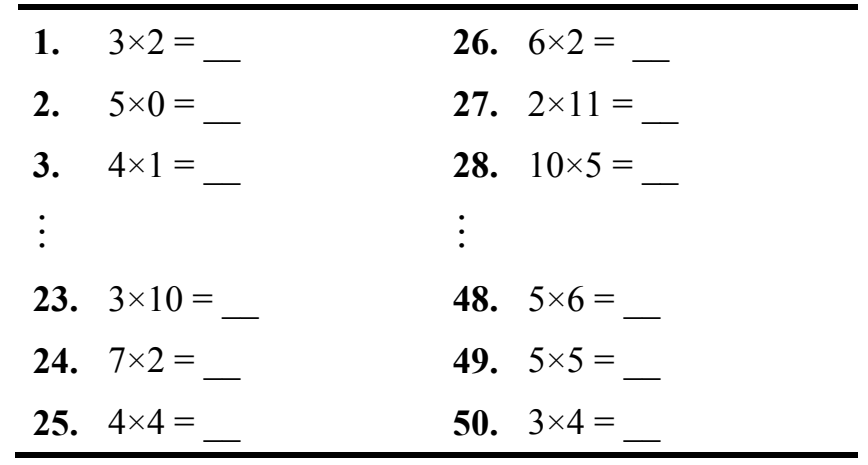

Figure 4: An abbreviated excerpt of commercially available multiplication tables

Such worksheets consist of columns of two digit multiplications where the answers have to be filled in. The levels of these sheets go from A through Z. A perfunctory glance at these sheets tells the reader that there is a strong focus on answers and perhaps no intellectual purpose or overall goal in terms of children 
filling in the sheets. The powerful influence of such commercial worksheets on the practice of teachers should not be underestimated. The mathematics imbedded in truly tabular multiplication tables escapes children, in addition to teachers.

As a true multiplication table, Figure 1 is a means to store or compress mathematics which can then be decompressed as length quantities in the case of rectangular areas on grid paper, for example. We now turn to a case where third grade students were taught their multiplication tables in such a way.

\section{When third grade students explore multiplication tables through rectangles}

During the early 2000s, I experimented with the idea of using Figure 1 as a means to teach the multiplication tables to a group of third grade students by using rectangles which they had to shade in on grid paper. This approach ties in with the two-dimensional layout of Figure 1, i.e. rows and columns.

The instruction to the third grade students was that they had to search for equivalences such as $2 \times 12=24$, $12 \times 2=24,3 \times 8=24,8 \times 3=24,4 \times 6=24$ and $6 \times 4=24$ in Figure 1 . These they then had to shade as rectangles on grid paper. These equivalences appear on the inside of Figure 1 in quite a structured way.

The students attended a school connected to a mathematics education project in a university town in the Midwest in the United States where I taught as a full time teacher. There was pressure from the school administration and parent community to teach the students their multiplication tables through rote memorisation. The way I went about examining the multiplication tables and two digit multiplication as elaborated so far, were thus not completely sanctioned by the school administration because it was found to be different. There was also no possibility of interviews with the students. The students' written work is therefore the main data source.

To do the multiplication as shown in the equivalences, I instructed the students to shade rows and columns of unit squares or blocks as they preferred to call them on the grid paper provided, to form rectangles. The grid paper was the centimetre paper as found in Burns (2000). For example, $3 \times 8=24$ meant shading a row of 3 blocks and a column of 8 blocks to form a rectangle that has 24 blocks. In other words, there is a connection between rows and columns and multiplier and multiplicand. This meant that they had to coordinate rows and columns in the way they had to do their shading. Such an action connected well with the two-dimensional format of the tabular multiplication table in Figure 1 which shows multiplier and multiplicand.

The equivalences referred to earlier are examples of the complex numerical information in a twodimensional form or layout typical of tables, as Robson (2003) points out. At the time, I became intrigued by the tabular formatting and especially the intercolumnar relationships, as revealed by the spread of the equivalences. Interestingly, they lie symmetrically with respect to the square numbers, e.g. 1, 4, 9, .. etc. They might well be considered as lists of multiplication facts when viewed outside the tabular multiplication table in Figure 1. Also, I hoped the students would recognise $8+8+8=24$ or $3+3+3+3+3+3=24$ as "repeated addition", as they coordinated their counting of shaded blocks in the rows and columns. In the end it was hoped that they would make a transition to multiplicative thinking from additive thinking. Admittedly there was nothing revolutionary about the instructing the third grade students how and what to do as they went about counting the blocks.

The two-dimensional form of the multiplication table in Figure 1 and its imbedded equivalences have conceptual connections with Steffe's $(1988 ; 1994)$ research on tracing third grade students' psychological structures for multiplication out of their structures for counting. For the students' multiplication schemes to emerge, they would have to think of a number such as 16 for example, through the notion of what Steffe calls composite units. A child who has formed composite units can understand the number 16 simultaneously as one group of sixteen and as sixteen individual units. A child's multiplying scheme involves the coordination of two composite units, which the tabular multiplication table (Figure 1) ought to foster in addition to my teaching. Sure enough there were cases where the students had the correct rectangles shaded on the grid paper in relation to the multiplication equivalences. Examples of work where students did not coordinate their shadings of the blocks or rectangles warrant our attention because they reveal a struggle in their transition from additive to multiplicative thinking despite my explicit instructions. 
The third grade student's work in Figure 5 shows her emergent multiplicative scheme. It comes from the case where a group of third grade students was asked to shade equivalences such as $2 \times 8=16,8 \times 2=16$, and $4 \times 4=16$.
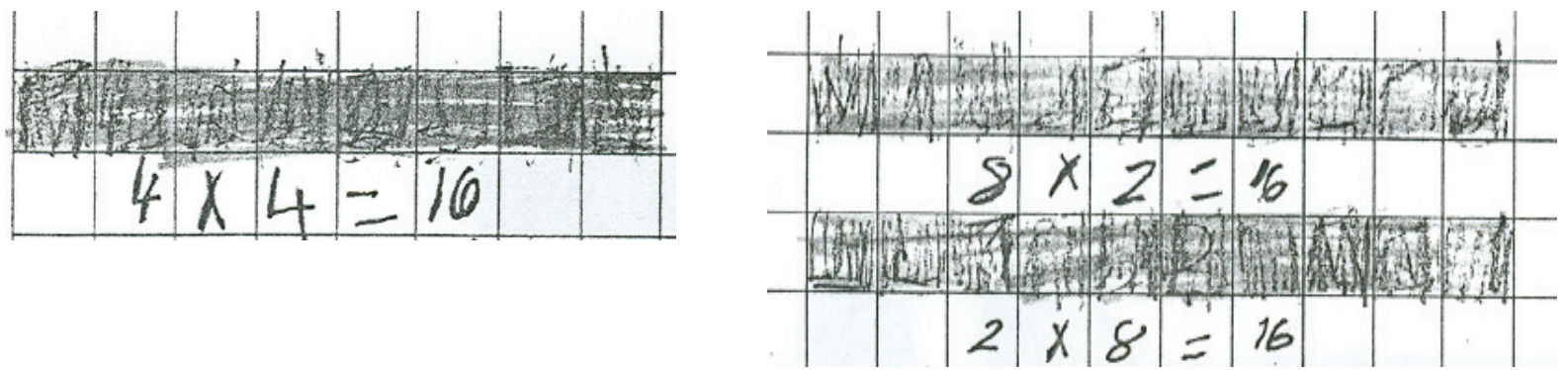

Figure 5: A third grade student's transition to multiplicative thinking

The third grade student's emergent multiplying scheme is related more to additive thinking in spite of the multiplication sign. For example, $4 \times 4=16$, represents counting $-4+4-$ which equals 8 . On the right side, the counting is $8+2(=10)$ and $2+8(=10)$. This third grade student's connection between multiplication and the underlying row and column structure of unit squares or blocks is not there. There was no coordination in the shading and in the counting of the blocks as composite units that yield 16 . What we see is what happened despite my instructions to the students on how to coordinate the rows and columns once equivalences have been identified inside Figure 1.

The finding illustrated here complements research that indicates that many students in the primary grade levels, whether they are in third or fifth grade, have trouble understanding the multiplicative relation between length and rectangular area measurement. For example, Izsák $(2004 ; 2005)$ found that fifth grade students, when representing rectangular areas on grid paper had problems because they had to "count the squares" or the blocks as in this case. His research was on how fifth grade students used their computational knowledge of whole number multiplication and connections between multiplication and discrete arrays to construct understandings of area.

Numbers in Figure 1 can be interpreted as composite units, counting schemes, and understandings of area. These are not the only possibilities with respect to Figure 1 . The table offers the potential for the gradual development of subject matter other than might naively be expected because of the numerical information presented in a two-dimensional, compact format. It is to this that we turn next.

\section{If these are multiplication tables, why are we doing addition ...?}

This question reflects a prospective teacher's reaction to my attempt to broaden thinking around the multiplication tables using Figure 1. The question came about when the following problem was posed to a class of prospective primary school teachers in one of the mathematics education modules that I taught:

Is there a way to find the sum of all the numbers in the $12 \times 12$ multiplication table, and can we extend our answer to an $n \times n$ multiplication table?

The fact that the multiplication tables, as represented in Figure 1 could be used for purposes other than multiplication was an unusual idea for the student, hence her question. My students had been made aware of the structure and symmetry in Figure 1 and were aware of its arithmetic. Even so, as revealed by this student's remark, they saw the arithmetic as fragmented, i.e. multiplication as separate from addition. My aim with the question was to seek alignment in my teaching with Learning Outcome 2, i.e. patterns, functions and algebra and mathematical skills such as specialising and generalising, pattern recognition and pattern extension. From previous class sessions, my students had been puzzled by the question on how one would introduce algebra in the middle grades.

From a historical perspective Figure 1 is a tabular representation in which arithmetical information is stored, recorded and where it can be retrieved. In particular, it can be used to investigate connections between arithmetic and algebra because of its two-dimensional format and imbedded multiplication sequences. Following Nunn (1919) we recognise no harsh dividing line between arithmetic and algebra: 
The difficulty of finding a precise difference between arithmetic and algebra (as these terms are commonly understood) is well known. It is due to the fact that the distinction between them consists not so much in a difference of subject-matter as in a difference of attitude towards the same subject matter. (p. 1)

The mathematical skills mentioned earlier play a key role in doing these investigations. For most of the prospective school teachers, the arithmetic in Figure 1 was obvious. It is to the problem posed to them earlier that we now return. The first row of the table below shows a shortened and generalised version of the numbers in Row 1 of Figure 1, i.e. the multiplication sequence 1, 2, $3 \ldots$

The last row in Figure 6 below is the sum of the consecutive multiplication sequence in Row 1 of Figure 1, i.e. the triangular numbers. The point is to find the functional relationship between the sum of a particular sequence in Row 1 and the indicated sum in the last row. Using equivalence, a particular relationship or pattern is recognised and extended, as indicated by the arrows. The middle rows, labeled multiply, show how this pattern is recognised by using equivalence. The last column is a generalisation of this pattern.

Through pattern recognition, pattern extension, specialising and generalising, it is possible to rewrite the arithmetical processes in the investigation to the point where the mathematical object emerges from the generalisation:

\begin{tabular}{lccccccccc}
\hline Row 1 of Figure 1 & $\mathbf{1}$ & $\mathbf{2}$ & $\mathbf{3}$ & $\mathbf{4}$ & $\mathbf{5}$ & $\mathbf{6}$ & $\ldots$ & $\boldsymbol{n}$ \\
\hline $\begin{array}{l}\text { Multiply } \\
\begin{array}{l}\text { Multiply } \\
\text { (equivalence) }\end{array}\end{array}$ & $\times 1$ & $\times 1 \frac{1}{2}$ & $\times 2$ & $\times 2 \frac{1}{2}$ & $\times 3$ & $\times 3 \frac{1}{2}$ & $\ldots$ & \\
$\begin{array}{l}\text { Multiply } \\
\text { (equivalence) }\end{array}$ & $\times \frac{2}{2}$ & $\times \frac{4}{2}$ & $\times \frac{5}{2}$ & $\times \frac{6}{2}$ & $\times \frac{7}{2}$ & $\ldots$ & \\
$\begin{array}{l}\text { Sum of Row 1 } \\
\text { (Triangular \#s) }\end{array}$ & $\mathbf{1}$ & $\times \frac{2+1}{2}$ & $\times \frac{3+1}{2}$ & $\times \frac{4+1}{2}$ & $\times \frac{5+1}{2}$ & $\times \frac{6+1}{2}$ & $\ldots$ & $\times \frac{n+1}{2}$ \\
\hline
\end{tabular}

Figure 6: Generating triangular numbers by summing the numbers in Row 1 in Figure 1

We would like a functional formula that will connect the numbers in the first row of Figure 6 with the last row as indicated by the various arrows. It is not always easy to find a function relationship (formula) by inspection, i.e. by just "looking" (Olivier, 1999, p. 2). By "filling in the gaps" such as writing $\times 1 \frac{1}{2}$ and $\times 2 \frac{1}{2}$ and the equivalences shown in the second row in Figure 6, we notice an emerging structure. The general case is arrived at by examining the special case of, for example, $5 \times 3=15$ as highlighted in Figure 6 .

In an inductive way we notice that the sum of the numbers in Row 1 of Figure 1 is:

$$
1+2+3+\ldots+12=\frac{12 \times(12+1)}{2}
$$

The sum for Row 2 therefore is:

$$
\begin{aligned}
& 2 \times(1+2+3+\ldots+12) \\
= & 2 \times\left(\frac{12 \times(12+1)}{2}\right)
\end{aligned}
$$

The sum for Row 3 therefore is:

$$
\begin{aligned}
& 3 \times(1+2+3+\ldots+12) \\
= & 3 \times\left(\frac{12 \times(12+1)}{2}\right)
\end{aligned}
$$


The sum for Row 12 is:

$$
\begin{aligned}
& 12 \times(1+2+3+\ldots+12) \\
= & 12 \times\left(\frac{12 \times(12+1)}{2}\right)
\end{aligned}
$$

The sum of all twelve rows therefore is:

$$
\begin{aligned}
& 1 \times\left(\frac{12 \times(12+1)}{2}\right)+2 \times\left(\frac{12 \times(12+1)}{2}\right)+3 \times\left(\frac{12 \times(12+1)}{2}\right)+\ldots+12 \times\left(\frac{12 \times(12+1)}{2}\right) \\
= & \left(\frac{12 \times(12+1)}{2}\right) \times(1+2+3+\ldots+12) \\
= & \left(\frac{12 \times(12+1)}{2}\right) \times\left(\frac{12 \times(12+1)}{2}\right) \\
= & \left(\frac{12(12+1)}{2}\right)^{2}
\end{aligned}
$$

Through induction we conclude that in an $n \times n$ tabular multiplication table as in Figure 1, the sum of the numbers is $\left(\frac{n(n+1)}{2}\right)^{2}$. The latter is a particular instance of reification where we end up with a mathematical object that captures particular arithmetical processes. In this way we have established a connection between arithmetic and algebra that stems from the multiplication table in Figure 1. In summary, it is difficult to claim that the object $\left(\frac{n(n+1)}{2}\right)^{2}$ is dangling in the air, disconnected from arithmetical processes, as we have seen so far. ${ }^{1}$

\section{Square numbers}

Next, we turn to the sequence of square numbers and investigate a way to find the sum of square numbers as shaded in the diagonal in Figure 7:

\begin{tabular}{|c|c|c|c|c|c|c|}
\hline 1 & 2 & 3 & 4 & 5 & 6 &. \\
\hline 2 & 4 & 6 & 8 & 10 & 12 &. \\
\hline 3 & 6 & 9 & 12 & 15 & 18 &. \\
\hline 4 & 8 & 12 & 16 & 20 & 24 &. \\
\hline 5 & 10 & 15 & 20 & 25 & 30 &. \\
\hline 6 & 12 & 18 & 24 & 30 & 36 &. \\
\hline. &. &. &. &. &. &. \\
\hline
\end{tabular}

Figure 7: The square numbers in Figure 1

This is in line with the prospective teacher's concern with why we were doing addition when we were focusing on the multiplication tables. The square numbers can be expressed by the object $n^{2}$. We thus pose the problem: How do we find the sum of sequence of square numbers, i.e. $1^{2}+2^{2}+3^{2}+\ldots+n^{2}$ ? It turns out the triangular numbers are helpful in terms of finding a functional relationship between the position of the square number and the sum of the square numbers, as illustrated in Figure 8.

${ }^{1}$ There are other interesting connections. For example, we know that $1^{3}+2^{3}+3^{3}+4^{3}+\ldots+n^{3}=\left(\frac{n(n+1)}{2}\right)^{2}$.

So we can confidently conjecture that the sum of the numbers in an $n \times n$ tabular multiplication table is $1^{3}+2^{3}+3^{3}+4^{3}+\ldots+n^{3}$. We leave it to the reader to "see" and explain $1^{3}+2^{3}+3^{3}+4^{3}+\ldots+n^{3}$ in Figure 1 ! And we also know that $1^{3}+2^{3}+3^{3}+4^{3}+\ldots+n^{3}=(1+2+3+4+\ldots+n)^{2}=\left(\frac{n(n+1)}{2}\right)^{2} \ldots$ 


\begin{tabular}{|c|c|c|c|c|c|}
\hline Position & 1 & $\ddots$ & 3 & $\ldots$ & $n$ \\
\hline $\begin{array}{l}\text { Triangular } \\
\text { numbers }\end{array}$ & 1 & 3 & 6 & & $\frac{n(n+1)}{2}$ \\
\hline Multiply & $\times 1$ & $\times \frac{5}{3}$ & $\times \frac{14}{6}$ & & \\
\hline $\begin{array}{l}\text { Multiply } \\
\text { (equivalence) }\end{array}$ & $\times \frac{3}{3}$ & $\times \frac{5}{3}$ & $\times \frac{7}{3}$ & & \\
\hline $\begin{array}{l}\text { Multiply } \\
\text { (equivalence) }\end{array}$ & $\times \frac{2 \times 1+1}{3}$ & $\times \frac{2 \times 2+1}{3}$ & $\times \frac{2 \times 3+1}{3}$ & & $\times \frac{2 n+1}{3}$ \\
\hline $\begin{array}{l}\text { Sum of diagonal } \\
\text { sequence }\end{array}$ & 1 & $5 \dot{\vdots}$ & 14 & & $\begin{array}{c}\frac{n(n+1)}{2} \times \frac{2 n+1}{3} \\
\text { Generalising }\end{array}$ \\
\hline
\end{tabular}

Figure 8: Generating a formula for summing the square numbers

By following the reasoning in Figure 8, we can establish a formula for the sum of the first $n$ square numbers:

$$
1^{2}+2^{2}+3^{2}+\ldots+n^{2}=\frac{n(n+1)}{2} \times \frac{2 n+1}{3}=\frac{n(n+1)(2 n+1)}{6}
$$

Further reification of arithmetical processes is possible if we ask how we can algebraify the sequence shown in the shaded diagonals symmetrical with respect to the square numbers in Figure 9:

\begin{tabular}{|c|c|c|c|c|c|c|}
\hline 1 & 2 & 3 & 4 & 5 & 6 &. \\
\hline 2 & 4 & 6 & 8 & 10 & 12 &. \\
\hline 3 & 6 & 9 & 12 & 15 & 18 &. \\
\hline 4 & 8 & 12 & 16 & 20 & 24 &. \\
\hline 5 & 10 & 15 & 20 & 25 & 30 &. \\
\hline 6 & 12 & 18 & 24 & 30 & 36 &. \\
\hline. &. &. &. &. &. &. \\
\hline
\end{tabular}

Figure 9: The sequence of numbers below and above the square numbers

\begin{tabular}{lcccccc}
\hline Position & $\mathbf{1}$ & $\mathbf{2}$ & $\mathbf{3}$ & $\mathbf{4}$ & $\boldsymbol{n}$ \\
\hline Multiply & $1 \times 2$ & $2 \times 3$ & $3 \times 4$ & $4 \times 5$ & $n \times(n+1)$ \\
Multiply (equivalence) & $1 \times(1+1)$ & $2 \times(2+1)$ & $3 \times(3+1)$ & $4 \times(4+1)$ & $n \times(n+1)$ \\
Diagonal sequence & $\mathbf{2}$ & $\mathbf{6}$ & $\mathbf{1 2}$ & $\mathbf{2 0}$ & $\begin{array}{c}n \times(n+1) \\
\text { Generalising }\end{array}$ \\
\hline
\end{tabular}

Figure 10: Generating a formula for sequence of numbers below and above the square numbers

The table in Figure 10 involves recognising, describing and representing patterns and relationships where eventually algebraic language and skills emerge.

Is there a way we can find an object for the sum of the multiplication sequence $2,6,12, \ldots$ as shown in the diagonal in Figure 9? What we are doing here is addition in the multiplication tables, which my students found as puzzling as the previous investigation. 


\begin{tabular}{lc:c:c:ccc}
\hline $\begin{array}{l}\text { Diagonal } \\
\text { sequence }\end{array}$ & 2 & 6 & 12 & 20 & $\ldots$ & $n \times(n+1) \vdots$ \\
$\begin{array}{l}\text { Sum of diagonal } \\
\text { sequence }\end{array}$ & 2 & 8 & & & & \\
\hline
\end{tabular}

Figure 11: Seeking a formula for the sum of diagonal sequences in Figure 9

What is the functional relationship between the variables in Figure 11, and how do we eventually generalise this relationship? From the previous exploration we noticed that the sequence of numbers in the shaded diagonal $2,6,12, \ldots$, which can be expressed as $n(n+1)$. After several attempts, we discover that the relationship is between the position of the sequence and its sum, multiplied by a triangular number, as is the case with the square numbers that we saw earlier:

\begin{tabular}{lccccc}
\hline Position & 1 & $\mathbf{2}$ & $\mathbf{3}$ & $\mathbf{4}$ & $\boldsymbol{n}$ \\
\hline Diagonal sequence & 2 & 6 & 12 & 20 & $n \times(n+1)$ \\
Multiply & $\times 1$ & $\times \frac{8}{6}$ & $\times \frac{20}{12}$ & $\times \frac{40}{20}$ & $?$ \\
$\begin{array}{l}\text { Multiply } \\
\text { (equivalence) }\end{array}$ & $\times \frac{6}{6}$ & $\times \frac{8}{6}$ & $\times \frac{10}{6}$ & $\times \frac{12}{6}$ & $?$ \\
$\begin{array}{l}\text { Multiply } \\
\text { (equivalence) }\end{array}$ & $\times \frac{2 \times 1+4}{6}$ & $\times \frac{2 \times 2+4}{6}$ & $\times \frac{2 \times 3+4}{6}$ & $\times \frac{2 \times 4+4}{6}$ & $\times \frac{2 \times n+4}{6}$ \\
$\begin{array}{l}\text { Sum of diagonal } \\
\text { sequence }\end{array}$ & $\mathbf{2}$ & $\mathbf{8}$ & $\mathbf{2 0}$ & $\mathbf{4 0}$ & $\frac{n \times(n+1) \times(2 n+4)}{6}$ \\
\hline
\end{tabular}

Figure 12: Seeking a formula for the sum of the diagonal sequence $2,8,20, \ldots$

The arithmetical processes are reified as the mathematical object $n(n+1) \frac{(2 n+4)}{6}$ in the lower right cell in Figure 12. Through similar processes of recognising patterns and generalising, the arithmetical processes can become mathematical objects for the next diagonal sequence and thus algebra. Figure 13 shows the different diagonal sequences, and related objects that describes their generalisations.

\begin{tabular}{lll}
\hline Diagonal sequence: Arithmetic & Mathematical objects: Algebra & Addition of diagonal sequence \\
\hline $1,4,9, \ldots$ & $n^{2}$ & $\frac{n(n+1)(2 n+1)}{6}$ \\
$2,6,12, \ldots$ & $n(n+1)$ & $\frac{n(n+1)(2 n+4)}{6}$ \\
$3,8,15, \ldots$ & $n(n+2)$ & $\frac{n(n+1)(2 n+7)}{6}$ \\
$4,10,18, \ldots$ & $n(n+3)$ & $\frac{n(n+1)(2 n+10)}{6}$ \\
$5,12,21, \ldots$ & $n(n+4)$ & $\frac{n(n+1)(2 n+13)}{6}$ \\
$\vdots$ & & \\
\hline
\end{tabular}

Figure 13: A partial list of arithmetic processes reified as algebraic objects 
A word of caution about the algebraic objects in Figure 13 is necessary. Sfard and Linchevski (1994, p. 191) point out that eventually all mathematical conceptions like these are endowed with a "processobject" duality. For example, the algebraic expression, say $3(x+5)+1$ may be interpreted in several different ways, for example:

0 it is a concise description of a computational process, a sequence of instructions: add 5 to the number at hand, multiply the result by 3 and add 1 .

0 it represent a certain number. It is a product of a computation rather than the computation itself. Even if this product cannot be specified at the moment because the number $x$ is presently unknown, it is still a number and the whole expression should be expected to behave like one.

$\circ$ it may be seen as a function - a mapping which translates every number $x$ into another. The expression does not represent any fixed (even if it is unknown) value. Rather it reflects a change, it is a function of two variables.

o it may be taken at its face value, as a mere string of symbols which represents nothing. It is an algebraic object in itself. Although semantically empty, the expression may still be manipulated and combined with other expressions according to certain well-defined rules.

What Sfard and Linchevski say about the expression $3(x+5)+1$ is applicable to the algebraic expressions in Figure 13.

What we have in Figure 13 are illustrations of how different numbers can be thought about and represented in various ways, according to Learning Outcome 1 and the emergence of algebra as generalised arithmetic, according to Learning Outcome 2 (DOE, 2003, pp. 7-9). We notice emerging mathematics that bridges obvious arithmetic - multiplication tables - and algebra. The reality is that, during most of my teaching, my students found the experiences of mathematical skills such as generalising, specialising, representing, pattern recognising and extending, and problem posing hard to follow, despite being told that we want to investigate ways of introducing algebra. One speculation is that their own schooling was never focused on such mathematical skills. Figure 13 is a summary of the compression or reification where we end up with mathematical objects in the column on the right hand side for each of the rows. On the other hand, it shows decompression of mathematical objects into arithmetical processes in the column on the left hand side in each of the rows.

\section{Implications for mathematics teacher education practice}

The "unpacking" and "compressing" of the rows, columns and diagonals in Figure 1 have implications for mathematics teacher education practice with its current focus on mathematics for teaching. In turn these are connected to the mathematical skills mentioned in the policy documents. As Adler (2005) notes unpacking and compressing are distinctive descriptions of the mathematical work that teachers do as they do their work, i.e. as they teach. How and where teachers should be afforded opportunities to learn mathematical skills that are centred on multiplication tables, which are after all, the mathematics in the foundation phase?

\section{The power of tables}

First, teachers should be provided with opportunities to comprehend the central message on the power of tables, starting with a historiography of tables per se. Robson's research informs us that during Babylonian times the tabular arrangements of the multiplication of numbers for the purposes of accounting and other metrological activities was a significant development. Additions and subtractions were carried out mentally or by other means and were never found on tablets. Surveyors needed multiplication, division and standardised constants for a number of tasks. This means that knowing your tables has to be viewed from a historical perspective because of the rich uses and opportunities that tables present us.

The ahistorical notion of tables is common to traditional knowledge assumptions in the mathematics curriculum and related teaching (Lampert, 1986a; 1986b). It is the expression of complex information in a two-dimensional form, as in Figure 1 that will forever elude us if we insist on narrow ways of thinking 
about tables. Such views of tables will never get us to envisage how arithmetical processes such as multiplication can be reified or compressed into mathematical objects commonly associated with algebra. Here are instances of packing or compressing where arithmetic becomes algebra.

In short, the complexity of the mathematics in Figure 1 is astonishing depending on the problems we pose regarding its rows and columns. It is the cognitive gains of the tabular format in Figure 1 that is striking. It all depends on the kinds of problems we pose in order to seek possible alignment with policy statements on mathematical skills and Learning Outcome 2 and/or Learning Outcome 1.

Separately listed multiplication tables, albeit well-organised, do not enable us to notice significant mathematics, as we have seen. It is the extended and deepened experience with tabular multiplication tables beyond "knowing one's tables" that is going to forge continuities in the highly fragmented mathematics curriculum. Thus a general observation is that tabulation needs to be reinstated into the multiplication tables in mathematics teacher education practice, which is one potential area where teachers can encounter tabulation.

This means that teachers should be exposed to multiplication table charts that exemplify tabulation where they can be provided with opportunities to search for structure and pattern, and in turn, compression and decompression. The mathematics that emerges within and across the rows and columns has a great deal to do with the practice of table making, which Robson and her colleagues have written so eloquently about. We pay a dear price if we ignore the technological and conceptual advances afforded by tabulation. Almost every time I have shown prospective and in-service teachers Figure 1, and pointed out that there is an astonishing amount of mathematics in it, I have received puzzled looks.

\section{Unpacking student work}

Second, teachers should have opportunities to unpack student work on multiplication tables such as that of the third grade student discussed earlier. The third grade students represented the multiplication tables in ways that departed from the well-known repeated addition model by focusing on rectangular area representations, which is intimately connected to the two-dimensional format in Figure 1. In their shading, they decompressed or represented multiplicative relationships in Figure 1 as rectangular areas. In doing so, they had to coordinate the shading of rows and columns in line with the two-dimensional tabular format common to tables per se.

The work conceptually connects multiplicative thinking and rectangular area. The third grade work, however, shows an intriguing mix of additive and multiplicative thinking despite my clear instructions. The correct rectangular area shading has deliberately not been shown. Even if it were, it is the finer details of their emergent multiplicative thinking that would be of interest and that teachers need to become familiar with as Steffe and Izsák alert us to.

We are reminded by Steffe $(1988$, p.136) that children "must not be forced to do things that they cannot do, such as learning their multiplication facts and algorithms for computation." For children multiplication is a compression of mathematics and also about composites, which they need to learn to represent in various ways, as suggested in Learning Outcome 1.

How and where would teachers have opportunities for learning to unpack such student work and the representations arising from multiplication tables? What would come in the way of teachers making sense of the rectangular areas or blocks that the third grade students shaded? I frequently hear teachers say something to the effect, but the children don't know their tables. The amount of curricular time spent on rote memorising the multiplication tables through having children fill in random lists of multiplication facts with its restricted focus on compressed, arithmetical representations underscores this point. These multiplication facts do not provide any fresh combination with the rest of the mathematics curriculum.

\section{The gains of compressing}

Third, there are irrefutable gains in reification or compressing, where the arithmetical processes at one level become mathematical objects at another level, leading to Figure 1 as generalised arithmetic, as advocated in the policy statements. These can be used to open teachers' eyes and ears to algebra, as 
Blanton and Kaput (2003) advocate. These are specific instances of "knowing mathematics for teaching." My prospective teachers have frequently been at a loss when they were asked to investigate or simply find the algebra in Figure 1. In particular, they experienced difficulty in following how equivalent numerical expressions lead to generalisation. These expressions play an important role in perceiving arithmetic as becoming algebra. Single multiplication lists or facts arranged like newspaper columns will enable teachers and students to notice the compression and decompression of arithmetical processes, such as we see thus far emerging from Figure 1, in a limited way. Sfard and Linchevski (1994) remind us that bridging the "gap" between arithmetic and algebra is a difficult one. It is the tabulation imbedded in Figure 1, accompanied by the use of mathematical skills that can make the transition from arithmetic to algebra more gradual not that there will be no cognitive difficulties along the way.

Compressing and decompressing the numbers in Figure 1 depend on problem posing one of the mathematical skills in the policy documents. Teachers and children encounter isolated multiplication tables very early in their schooling, implying that making connections to other branches of mathematics becomes difficult. The one prospective teacher's reaction, "if these are the multiplication tables, why are we doing addition," comes to mind. This student was baffled by the idea of wanting to add up the numbers in Figure 1.

In summary, how and where will teachers be afforded with opportunities to pose problems on the numbers in Figure 1 in various ways, as written in the policy document in Learning Outcome 1 which can lead to Learning Outcome 2? Teachers and people in general, need to know the reductive status of the multiplication tables in the mathematics curriculum and hence our inability to foster epistemic openness and interpretive possibilities with respect to number. This status makes us unable to notice mathematics beyond multiplication in Figure 1. One of children's first rote memorisation experiences in school is when they have to know their multiplication tables in sequence, as was a common practice in Babylonia according to Robson's research. If we wish to understand multiplication we must first understand multiplicative thinking as an instance of epistemic openness and how surprisingly slowly it develops in children (Clark \& Kamii, 1996). Like the prospective teachers, I have been drilled in a rote memorisation of multiplication lists, probably in similar ways to Mesopotamian scholastic mathematics but definitely not in the same volume. Robson (2003, p. 42) notes that at that time there was a preference for arithmetical and metrological equivalences as lists. Most children in school are surrounded by wall charts in the form of lists of multiplication facts. She cautions us that we pay a heavy price as a result of our collective blindness to the power of tabular formatting such as we find in Figure 1. It is this formatting that I wanted my third grade students and currently, my prospective teachers to notice. Observing the structure in Figure 1 and the problems we pose with respect to the structure, is what we need to accomplish. Modal multiplication tables practice in schools is not going to get us there.

\section{Conclusion}

There is no doubt that the mathematics that comes about in this paper is dependent on the teaching practices associated with the two-dimensional format of the multiplication table in Figure 1. Any severance between the mathematics and teaching practices will not enable us to perceive the depth and richness of the problems we posed and investigated, starting from the case of the third grade students to the emergence of algebra at higher grade levels.

It must be noted that this paper is doubtless flawed and may even be misguided when it questions standardised tests and teaching practices that emphasise rote recall of multiplication facts and exercises where children's ability to perform multi-digit computation problems by relying on a rote memorisation of the so-called multiplication tables. However, such teaching practices rarely arrive at the underlying structure in the tabular multiplication table we started out with. Also, they are not aligned with policy statements on the mathematical skills illustrated in this paper that are crucial to knowing mathematics for teaching.

It is hoped that something worthwhile can be learned from the close probing of the history of tables and in turn, a truly tabular multiplication table such as Figure 1. Knowing multiplication tables for the purpose of computations has an important but limiting role. It is, however, the table making and tabulation processes that we inherited from the Babylonians and later civilisations that allow us to revisit a simple 
multiplication table such as Figure 1 to search for the algebra in it and see other mathematics such as those of the third grade student.

The narrow floodlight of knowing one's table is mostly confined to computation. Echoing Robson, today we can bring into the open sunlight our understanding of multiplication tables to a point where we can uncover structure, abstraction, and algebra through compression and decompression, whether it is in the elementary (primary) or high school. We are in need of these and other fresh combinations.

\section{References}

Adler J. (2005). Mathematics for teaching: What is it and why we need to talk about it? Pythagoras, 62, 5-11.

Adler J., Ball, D. L., Krainer, K., Lin, F-L., \& Novotna, J. (2005). Reflections on an emerging field: Researching mathematics teacher education, Educational Studies in Mathematics 60, 359-381.

Ball, D. L., \& Bass, H. (2000). Interweaving content and pedagogy in teaching and learning to teach: Knowing and using mathematics. In J. Boaler (Ed.), Multiple perspectives in mathematics teaching and learning (pp. 83-104).Wesport, CT: Ablex Publishing.

Ball, D. L., Bass, H., \& Hill, H. (2004). Knowing and using mathematical knowledge in teaching: Learning what matters. In A. Buffler \& R. Lausch (Eds.), Proceedings of the $12^{\text {th }}$ Annual Conference of the Southern African Association for Research in Mathematics, Science and Technology Education (pp. 51-56). Durban: SAARMSTE.

Blanton, M. L., \& Kaput, J. (2003). Developing elementary teachers' 'Algebra eyes and ears'. Teaching Children Mathematics 10(2), 70-77.

Brown, S., \& Walter, M. I. (1990). The art of problem-posing (2nd ed.). Hillsdale, NJ: Lawrence Erlbaum Associates.

Burns, M. (2000). About mathematics teaching: A K-8 resource. Sausalito, CA: Math Solutions Publications.

Clark, F. B., \& Kamii, C. (1996). Identification of multiplicative thinking in children in grades 1-5, Journal for Research in Mathematics Education, 27(1), 41-51.

Department of Education (DOE). (2003). Revised national curriculum statement: Learning area statement for mathematics. Pretoria: Department of Education.

Dewey, J. (1964). The relation of theory to practice in education. In R. Archambault (Ed.), John Dewey on education. Chicago: University of Chicago Press. (Original work published in 1904)

Izsák, A. (2004). Teaching and learning two-digit multiplication: Coordinating analyses of classroom practices and individual student learning. Mathematical Thinking and Learning, 6(1), 37-79.

Izsák, A. (2005). 'You have to count the squares': Applying knowledge in pieces to learning rectangular area. The Journal of the Learning Sciences, 14(3), 361-403.

Lampert, M. (1986a). Knowing, doing, and teaching multiplication. Cognition and Instruction, 3, 305-342.

Lampert, M. (1986b). Teaching multiplication. Journal of Mathematical Behavior, 5, 241-280.

Mason, J. H. (1998). Learning and doing mathematics (2nd revised ed.). York: QED Books.

Nunn, T. P. (1919). The teaching of algebra. London: Longmans, Green and Company.

Olivier, A. (1999). Circles, regions and chords. Retrieved May 24, 2009, from http://www.sun.ac.za/mathed/ 174/CirclesRegionsChords.pdf.

Olivier, A. (2002). Aspects of mathematical knowledge for teaching. Analysing mathematical thinking: A problem-centred approach. Retrieved December 12, 2007, from http://www.sun.ac.za/mathed/174/ InduksieDeduksie.pdf.

Robson, E. (1998). Counting in cuneiform, Mathematics in Schools, 27(4), 2-9.

Robson, E. (2000). Mesopotamian mathematics: some historical background. In V. J. Katz (Ed.), Using history to teach mathematics (pp. 149-158). Washington, DC: Mathematical Association of America.

Robson, E. (2002). More than metrology: Mathematics education in an Old Babylonian scribal school. In J. M. Steele \& A. Imhausen (Eds.), Under one sky: Mathematics and astronomy in the ancient Near East (Alter Orient und Altes Testament, 297) (pp. 325-365). Münster: Agarit-Verlag.

Robson, E. (2003). Tables and tabular formatting in Sumer, Babylonia, and Assyria, 2500-50 BCE. In M. Campbell-Kelly, M. Croarken, R. G. Flood, \& E. Robson (Eds.), The history of mathematical tables from Sumer to spreadsheets (pp. 18-47). Oxford: Oxford University Press.

Robson, E. (2004). Accounting for change: The development of tabular bookkeeping in early Mesopotamia. In M. Hudson \& C. Wunsch (Eds.), Creating economic order: Record-keeping, standardization, and the development of accounting in the Ancient Near East (pp. 107-144). Bethesda, MD: CDL Press. 
Robson, E. (2007). Mathematics, metrology, and professional numeracy, In G. Leick (Ed.), The Babylonian world (pp. 414-427). London: Routledge.

Sfard, A., \& Linchevski, L. (1994). The gains and the pitfalls of reification: The case of algebra. Educational Studies in Mathematics, 26, 191-228.

Steffe, L. P. (1988). Children's construction of number sequences and multiplying schemes. In J. Hiebert \& M. Behr (Eds.), Number concepts and operations in middle grades (pp. 119-140). Reston, VA: National Council of Teachers of Mathematics.

Steffe, L. P. (1994). Children's multiplying schemes. In G. Harel \& J. Confrey (Eds.), The development of multiplicative reasoning in the learning of mathematics (pp. 3-39). Albany: State University of New York Press.

Whitehead, A. N. (1929/1951). The aims of education and other essays. New York: Macmillan. 